\title{
Avaliação da Frequêencia Cardíaca Embriofetal no Primeiro Trimestre da Gestação por meio da Ultra-sonografia Transvaginal com Doppler Colorido e Pulsátil
}

\author{
Assessment of Embryo Heart Rate in Early Pregnancy by Transvaginal \\ Ultrasonography with Color and Pulsed Doppler
}

Cláudia Lemos da Silva, Luiz Antônio Bailão

\begin{abstract}
RESUMO
Objetivos: avaliar a evolução da freqüencia cardiaca embrio-fetal no primeiro trimestre da gestação e determinar sua curva de normalidade.

Pacientes e Métodos: em estudo prospectivo foram avaliadas 206 pacientes com diagnóstico clínico e/ ou ultra-sonográfico de gestação no primeiro trimestre, por meio de exame ultrasonográfico transvaginal com Doppler colorido, utilizando-se equipamento da marca Aloka, modelo SSD-2000, com sonda transvaginal convexa na freqüência de 5,0 MHz, todos realizados por um mesmo examinador. Foi determinada a freqüencia cardiaca embriofetal. As pacientes foram divididas em grupos de acordo com a idade gestacional, em intervalos de 0,5 semana a partir da $6^{a}$ semana de gestação. Foi avaliada a evolução da gestação mediante realização de exame ultra-sonográfico de rotina no final do $2^{\circ}$ e $3^{\circ}$ trimestre. Foram calculados as médias e desvios-padrão para cada idade gestacional avaliada.

Resultados: foi possivel determinar a curva de normalidade para a freqüência cardiaca embriofetal. A média da freqüência cardiaca embriofetal apresentou modificações com a evolução da idade gestacional, variando de $110 \pm 14$ bpm com 6,0 semanas a $150 \pm 12$ bpm com 14,0 semanas, compativeis com as fases do desenvolvimento e maturação funcional cardiaca.

Conclusões: a ultra-sonografia transvaginal com Doppler colorido tornou possivel a avaliação cardiovascular da gestação inicial, sendo um método não-invasivo e inócuo para o embrião. Os valores determinados poderão ser utilizados em estudos futuros neste periodo gestacional.
\end{abstract}

PALAVRAS-CHAVE: Ultra-sonografia. Dopplervelocimetria. Freqüência cardiaca fetal. Gravidez normal.

\section{Introdução}

A introdução do Doppler em sondas transvaginais abriu novas perspectivas ao exame do embrião, permitindo melhores estudos sobre a anatomia vascular e hemodinâmica embrionária ${ }^{1}$. Esta associação permitiu também uma importante redução no tempo de realiza-

Departamento de Ginecologia e Obstetrícia do Hospital das Clinicas da Faculdade de Medicina de Ribeirão Preto - USP; Diagnosis - Centro de Diagnóstico em Medicina - Ribeirão Preto.

Correspondência:

Cláudia Lemos da Silva

R. XV de Novembro, 363, Ap 1800

38400-214 - Uberlândia - MG ção do exame, diminuindo assim a exposição do embrião ao efeito do Doppler e, conseqüentemente, tornando seu uso de baixo risco na gestação inicial ${ }^{2}$.

O sistema cardiovascular é o primeiro a funcionar no embrião, com o coração começando a contrair-se 22 dias após a concepção ( 5 semanas e 1 dia após o último periodo menstrual), apresentando inicialmente uma motilidade ondulatória de tipo peristáltico. A freqüência cardíaca aumenta inicialmente devido ao desenvolvimento das câmaras cardíacas e ao automatismo específico intrínseco. Primeiro o ritmo é de origem ventricular e assim que as aurículas se desenvolvem assumem a função de marcapasso, 
aumentando a freqüência dos movimentos ${ }^{1}$. A maturação do sistema condutor condiciona o aumento da freqüência cardiaca ${ }^{3}$, a qual diminui posteriormente devido à maturação funcional do sistema parassimpático, à expansão do leito vascular e ao estabelecimento de conexões secundárias entre os vasos coriônicos, vitelínicos, umbilicais e embrionários ${ }^{4}$. $\mathrm{O}$ controle neurogênico imaturo da freqüência cardiaca embrionária pode explicar as pequenas variações encontradas antes da décima semana de gestação, contrastando com a atividade neurogênica autonômica mais dominante esperada após este período. A freqüência cardíaca mantém-se mais ou menos constante ao longo do segundo e terceiro trimestres da gestação ${ }^{1}$.

Goldstein ${ }^{5}$, com a ultra-sonografia transvaginal, detectou a presença de atividade cardiaca em todos os embriões com comprimento crânionádega $(\mathrm{CCN})$ maior ou igual a $4 \mathrm{~mm}$ (6 semanas), sugerindo que a ausência de batimento cardíaco detectável pela ultra-sonografia transvaginal em embriões com $\mathrm{CCN}$ maiores ou iguais a $5 \mathrm{~mm}(6,5$ semanas) pode ser compativel com gestação inviável.

Robinson e Shaw-Dunn ${ }^{6}$ foram os primeiros a observar um aumento da freqüência cardíaca embrionária de 123 batimentos por minuto (bpm) na metade da sexta semana gestacional para $177 \mathrm{bpm}$ na nona semana. Shenker et al. ${ }^{7}$ observaram que a freqüência cardiaca embrionária aumentou de uma média de $90 \mathrm{bpm}$ na sexta semana para uma média de $134 \mathrm{bpm}$ na sétima semana. Cadkin e McAlpin ${ }^{8}$ descreveram um progressivo aumento na média da freqüência cardiaca embrionária de 100-110 bpm na sexta semana para $144 \mathrm{bpm}$ na nona semana. Achiron et al. ${ }^{9}$, determinaram a construção de normogramas para a freqüência cardiaca embrionária entre 5,5 e 11 semanas de gestação, em que a freqüência cardiaca aumentou progressivamente de $110 \mathrm{bpm}$ entre 5,5 e 6 semanas para $171-178 \mathrm{bpm}$ entre 8 a 10 semanas, permanecendo estável em $170 \mathrm{bpm}$ após 10 semanas, tendo seus desvios da normalidade correlação positiva com perda embrionária.

Laboda et al. ${ }^{10}$ foram pioneiros ao correlacionar evolução gestacional anormal com a freqüência cardiaca embrionária, observando que freqüências abaixo de $85 \mathrm{bpm}$ (-2DP) apresentaram correlação positiva com perda gestacional. Achiron et al. ${ }^{9}$ sugerem também que abortamento espontâneo no primeiro trimestre pode ser previsto por medida anormal da freqüência cardiaca embrionária. Schats et al. $^{3}$ demonstraram que quanto mais tardio o aparecimento de atividade cardiaca, maior a chance de perda gestacional.
A freqüência cardíaca embrionária precedendo a perda gestacional pode ser normal ou baixa, mas acaba caindo abaixo da curva de referência. Este padrão de desvio parece refletir uma redução na capacidade de crescimento do embrião ${ }^{11}$, a qual mais tarde poderá resultar em aborto espontâneo. A freqüência cardíaca acima de $200 \mathrm{bpm}$ pode refletir ainda a presença de infecção concomitante ${ }^{9}$. Doubilet e Benson ${ }^{12}$ relataram que freqüência cardíaca embrionária abaixo dos limites da normalidade para determinada idade gestacional pode indicar mau prognóstico gestacional. Antes da décima semana de gestação a freqüência cardíaca é tida como capaz de prever perda gestacional ${ }^{10,13,14}$, devendo portanto ser avaliada previamente em embriões que serão submetidos a biópsia de vilosidades coriônicas para estudo citogenético.

Em estudo recente, Hyett et al. ${ }^{15}$ demonstraram a importância de se incluir a medida da freqüência cardiaca como parte da rotina de avaliação fetal nos primeiros meses de gestação: ela não somente contribui para a suspeita de anomalia cromossômica fetal (bradicardia na trissomia 18 ou taquicardia na trissomia 21), mas também aumenta a sensibilidade do "screening" pela idade materna e espessura da translucência nucal em predizer o risco para anomalias cromossômicas.

O objetivo deste estudo foi determinar uma curva de normalidade da freqüência cardiaca embriofetal no primeiro trimestre gestacional que possa ser utilizada para auxiliar na determinação do prognóstico gestacional.

\section{Pacientes e Métodos}

Foi realizado estudo transversal no qual foram incluidas 323 mulheres, voluntárias, com diagnóstico clínico, laboratorial e/ou ultra-sonográfico de gestação no primeiro trimestre (5 a 14 semanas). Estas pacientes foram atendidas no Ambulatório de Pré-natal do Hospital das Clínicas de Ribeirão Preto ou na rede pública municipal e encaminhadas para avaliação ultrasonográfica no período de 1 de fevereiro de 1995 a 1 de fevereiro de 1997. O estudo foi aprovado pela Comissão de Normas Éticas e Regulamentares da Instituição Hospital das Clínicas de Ribeirão Preto.

Foram excluídas do estudo cento e treze pacientes por falta aos retornos agendados, manifestação de doenças clínicas (hipertensão arterial crônica, nefropatia, cardiopatia), doenças ginecológicas no exame inicial (abortamento ha- 
bitual, miomatose uterina, presença de DIU), casos de gestação múltipla, intercorrências obstétricas no exame inicial (abortamento inevitável, completo, incompleto, retido, ovo anembrionado), tabagismo ou ainda ausência de fluxo diastólico na artéria uterina no exame inicial. Pacientes com sangramento vaginal não foram excluídas devido à alta freqüência de sangramento de implantação verificada neste periodo da gestação, caracterizado à ultra-sonografia pela ausência de achados ultra-sonográficos anormais e embrião com vitalidade preservada.

As pacientes com idade gestacional entre 4,5 e 5,5 semanas $(n=4)$ foram excluídas na análise dos resultados posteriormente, em razão do pequeno número de pacientes em cada um destes grupos, restando 206 pacientes para análise final, com distribuição da idade gestacional apresentada na Tabela 1.

Tabela 1 - Distribuição das pacientes por idade gestacional em semanas.

\begin{tabular}{cc}
\hline Idade gestacional & $\mathbf{n}$ \\
\hline incipiente & $2^{*}$ \\
4,5 & $1^{*}$ \\
5,0 & $1^{*}$ \\
5,5 & 0 \\
6,0 & 5 \\
6,5 & 5 \\
7,0 & 8 \\
7,5 & 10 \\
8,0 & 8 \\
8,5 & 13 \\
9,0 & 17 \\
9,5 & 18 \\
10,0 & 13 \\
10,5 & 17 \\
11,0 & 16 \\
11,5 & 12 \\
12,0 & 18 \\
12,5 & 16 \\
13,0 & 14 \\
13,5 & 5 \\
14,0 & 11 \\
Total & $210^{*}$ \\
\hline
\end{tabular}

*4 pacientes com idade gestacional inferior a 5,5 semanas foram excluídas.

As pacientes foram avaliadas pelo mesmo examinador em decúbito dorsal, em posição ginecológica, após esvaziamento vesical, com du- ração do exame variando de 5 a 10 minutos. O equipamento utilizado foi da marca Aloka, modelo SSD-2000, com sonda transvaginal convexa com freqüência de emissão de $5,0 \mathrm{MHz}$ e o filtro fixado em $50 \mathrm{~Hz}$.

A idade gestacional foi calculada por meio da data da última menstruação e confirmada pela medida do comprimento do embrião $(\mathrm{CCN})$, até a décima segunda semana ou diâmetro biparietal (DBP) e circunferência craniana (CC) da décima segunda à décima quarta semana. As pacientes foram divididas em grupos de acordo com a idade gestacional, em intervalos de meia semana. A freqüência cardíaca embrionária foi determinada em todos os casos após a identificação do embrião, a partir da sexta semana de gestação, mediante localização dos batimentos cardiacos pelo Doppler colorido e traçado com Doppler pulsátil, com cálculo realizado pelo próprio equipamento por cáliper fixado em intervalo de tempo entre dois ciclos cardiacos consecutivos.

A avaliação da evolução da gestação foi feita pela realização de exame ultra-sonográfico convencional no final do segundo e terceiro trimestre, realizados pelo mesmo examinador, segundo protocolo pré-determinado. Foi avaliado o resultado pós-natal por meio dos dados obtidos dos recém-nascidos, por dados fornecidos pelas pacientes ou por investigação diretamente nos prontuários dos hospitais da rede pública.

Os parâmetros numéricos foram avaliados pelo programa Microsoft Excel (Microsoft Corp. ${ }^{\circledR}$ ), versão 7.0, calculando-se média, mediana, moda e desvio padrão para cada item. Calculou-se ainda o índice de assimetria (média - moda/desviopadrão) e o coeficiente de variabilidade de Pearson [(desvio-padrão/média) x 100] para determinação de padrão gaussiano (distribuição normal)

\section{Resultados}

Foi avaliada a freqüência cardíaca embriofetal no primeiro trimestre gestacional, entre a sexta e a décima quarta semana, em intervalos de meia semana. Os valores médios encontrados estão representados na Tabela 2 e na Figura 1.

Observou-se um aumento progressivo da freqüência cardíaca a partir da sexta semana até a nona semana (110 $\pm 14 \mathrm{bpm})$, estabilizando-se entre 9,5 e 11,5 semanas (média de $165 \mathrm{bpm}$ ), sofrendo pequena queda a partir de então até a décima quarta semana $(150 \pm 12 \mathrm{bpm})$.

A evolução da gestação foi normal em 184 
$(89,3 \%)$ pacientes. As demais apresentaram como intercorrências: DHEG leve (6 pacientes), macrossomia (5 pacientes), oligo-hidrâmnio (2 pacientes), malformação fetal com óbito (2 pacientes), abortamento ( 2 pacientes), amniorrexe prematura (1 paciente), espessamento placentário (1 paciente), aceleração da maturidade placentária (1 paciente), infecção fetal (1 paciente) e RCIU (1 paciente).

Tabela 2 - Médias e desvios-padrão da freqüência cardíaca embrio-fetal em função da idade gestacional em 206 grávidas normais.

\begin{tabular}{crccc}
\hline $\begin{array}{c}\text { Idade gestacional } \\
\text { (semanas) }\end{array}$ & $\mathbf{n}$ & \multicolumn{3}{c}{ Freqüência cardíaca (bpm) } \\
\hline 6,0 & 5 & $\mathbf{- 2 D P}$ & $\mathbf{M}$ & +2DP \\
6,5 & 5 & 96 & 110 & 124 \\
7,0 & 8 & 113 & 124 & 143 \\
7,5 & 10 & 136 & 152 & 157 \\
8,0 & 8 & 148 & 162 & 168 \\
8,5 & 13 & 158 & 170 & 183 \\
9,0 & 17 & 164 & 175 & 186 \\
9,5 & 18 & 155 & 173 & 191 \\
10,0 & 13 & 151 & 165 & 179 \\
10,5 & 17 & 145 & 166 & 186 \\
11,0 & 16 & 151 & 166 & 182 \\
11,5 & 12 & 157 & 167 & 177 \\
12,0 & 18 & 143 & 160 & 176 \\
12,5 & 16 & 143 & 158 & 173 \\
13,0 & 14 & 145 & 156 & 167 \\
13,5 & 5 & 140 & 152 & 164 \\
14,0 & 11 & 138 & 150 & 162 \\
\hline n: número de pacientes & M: média & DP: desvio-padrão & & \\
& & & &
\end{tabular}

\section{Discussão}

Os equipamentos ultra-sonográficos de alta resolução, particularmente com o emprego da sonda transvaginal, proporcionam condições suficientes para detectar sinal cardiaco antes que a estrutura embrionária esteja distinta da vesícula vitelínica $^{5}$. A atividade cardiaca foi detectada em todos os embriões com $4 \mathrm{~mm}$ ou mais de CCN, concluindo-se que ausência de batimentos cardiacos detectáveis pela ultra-sonografia transvaginal em embriões de $5 \mathrm{~mm}$ ou mais sugere uma gestação inviável. A ausência de atividade cardíaca em um embrião de $3 \mathrm{~mm}$ ou menos necessita de uma reavaliação ultra-sonográfica em 3 a 5 dias, pois o crescimento embrionário estimado é de cerca de $1 \mathrm{~mm}$ por dia em embriões normais. $\mathrm{O}$ menor embrião com atividade cardíaca visivel neste estudo apresentou-se com CCN de $3 \mathrm{~mm}$.

Observou-se um aumento da freqüência cardíaca da sexta até a nona semana, com valores médios de 110 e $175 \mathrm{bpm}$, respectivamente, estabilizando-se entre a metade da nona semana e a metade da décima primeira semana (média de $166 \mathrm{bpm})$, para diminuir em seguida até a décima quarta semana (média de $150 \mathrm{bpm}$ ), compativeis com o estudo de Achiron et al. ${ }^{9}$, porém com valores médios pouco diferentes dos encontrados pelos demais autores. As variações da freqüência cardiaca com o aumento da idade gestacional foram compativeis com as fases do desenvolvimento e maturação funcional cardiaca.

Concluindo, a ultra-sonografia transvaginal com Doppler colorido tornou possivel a avaliação cardíaca da gestação inicial, sendo um método não-invasivo, inócuo para o embrião e de ampla aplicação neste período gestacional.

Os valores determinados poderão ser utilizados em novos estudos no sentido de avaliar o risco de perda gestacional, associado ou não a patologias do primeiro trimestre da gestação.

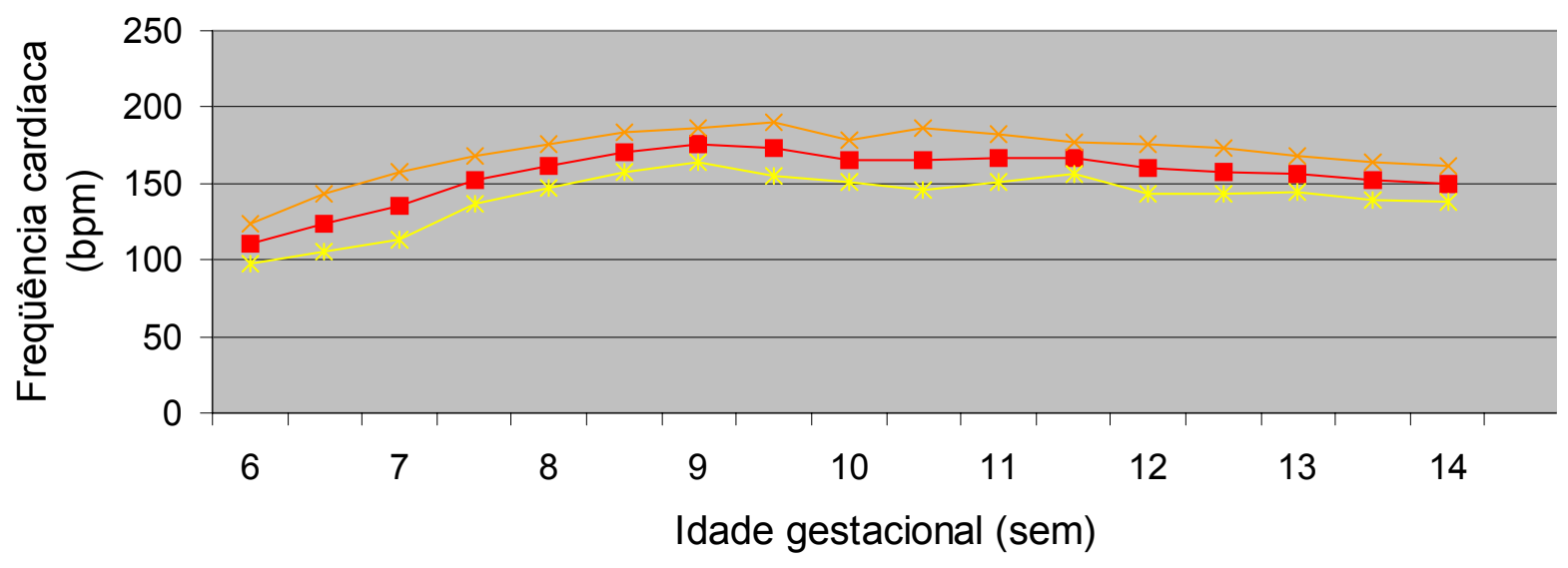

Figura 1 - Representação gráfica das médias e desvios-padrão da freqüência cardíaca embriofetal em função da idade gestacional em 206 grávidas normais. 


\section{SUMMARY}

Purpose: to evaluate the evolution in the embryo heart rate in the first trimester of pregnancy.

Patients and Methods: in a prospective study 206 pregnant women were evaluated in the first trimester of pregnancy, by transvaginal color Doppler sonography, using Aloka, SSD-2000 apparatus, with a 5-MHz transvaginal transducer. All examinations were performed by the same examiner, with the determination of embryo heart rate. The patients were classified into groups according to the gestational age, in half-week intervals from the 5 th week of pregnancy on. Pregnancy outcome was evaluated by ultrasonography at the end of second and third trimesters. Mean and standard deviation were determined for each evaluated gestational age.

Results: it was possible to determine normal values for embryo heart rate. Mean embryo heart rate showed changes with gestational age, ranging from $110 \pm 14 \mathrm{bpm}$ at the 6.0th week to $150 \pm 12 \mathrm{bpm}$ at the 14.0th week. Conclusions: transvaginal pulsed color Doppler equipment enabled cardiovascular evaluation in early pregnancy, being a noninvasive method and innocuous to the embryo. These values would be useful in new studies on dopplervelocimetry in this period of pregnancy.

KEY WORDS: Ultrasonography. Dopplervelocimetry. Fetal heart rate. Normal pregnancy.

\section{Referências}

1. Montenegro N, Areias JC, Leite LP. Endosonografia e doppler transvaginal na avaliação hemodinâmica embrio-fetal no $1^{\circ}$ trimestre da gravidez. Acta Med Port 1993; 6 Suppl 1:13-8.

2. Dillon EH, Case CQ, Ramos IM, Holland CK, Taylor KJ. Endovaginal pulsed and color Doppler in first trimester pregnancy. Ultrasound Med Biol 1993; 19:517-25.
3. Schats R, Jansen CA, Wladimiroff JW. Embryonic heart activity: appearance and development in early human pregnancy. Br J Obstet Gynaecol 1990; 97:989-94.

4. Montenegro N, Ramos C, Matias A, Barros H. Variation of embryonic/fetal heart rate at 6-13 weeks' gestation. Ultrasound Obstet Gynecol 1998; 11:274-6.

5. Goldstein SR. Significance of cardiac activity on endovaginal ultrasound in very early embryos. Obstet Gynecol 1992; 80:670-2.

6. Robinson HP, Shaw-Dunn J. Fetal heart rates as determined by sonar in early pregnancy. J Obstet Gynaecol Br Commonw 1973; 80:805-9.

7. Shenker L, Astle C, Reed K, Anderson C. Embryonic heart rates before the seventh week of pregnancy. J Reprod Med 1986; 31:333-5.

8. Cadkin AV, McAlpin J. Detection of fetal cardiac activity between 41 and 43 days of gestation. J Ultrasound Med 1984; 3:499-503.

9. Achiron R, Tadmor O, Mashiach S. Heart rate as a predictor of first trimester spontaneous abortion after ultrasound proven viability. Obstet Gynecol $1991 ; 78: 330-4$.

10.Laboda LA, Estroff JA, Benacerraf BR. First trimester bradycardia: a sign of impeding fetal loss. J Ultrasound Med 1989; 8:561-3.

11.Schats R. Embryonic cardiac activity: appearance and development in early human pregnancy. Med Rev 1993; 44:42-6.

12.Doubilet PM, Benson CB. Embryonic heart rate in the early first trimester: what rate is normal? $\mathrm{J}$ Ultrasound Med 1995; 14:431-4.

13. May DA, Sturtevant NV. Embryonical heart rate as a predictor of pregnancy outcome: a prospective analysis. J Ultrasound Med 1991; 10:591-3.

14.Merchiers EH, Dhont M, De Sutter PA, Beghin CJ, Vandekerckhove DA. Predictive value of early embryonic cardiac activity for pregnancy outcome. Am J Obstet Gynecol 1991; 165:11-4.

15. Hyett JA, Noble PL, Snijders RJM, Montenegro N, Nicolaides $\mathrm{KH}$. Fetal heart rate in trisomy 21 and other chromosomal abnormalities at 10-14 weeks of gestation. Ultrasound Obstet Gynecol 1996; 7:239-44.

\section{FAÇA UMA VISITA A HOME PAGE DA FEBRASGO www.febrasgo.org.br}

